\title{
New Results on Passivity for Discrete-Time Stochastic Neural Networks with Time-Varying Delays
}

\author{
Wei Kang, ${ }^{1,2}$ Jun Cheng, ${ }^{3}$ and Xiangyang Cheng ${ }^{4}$ \\ ${ }^{1}$ School of Mathematical Sciences, University of Electronic Science and Technology of China, Chengdu 611731, China \\ ${ }^{2}$ School of Information Engineering, Fuyang Teachers College, Fuyang 236041, China \\ ${ }^{3}$ School of Electrical and Information Technology, Yunnan Minzu University, Kunming 650500, China \\ ${ }^{4}$ School of Business, Fuyang Teachers College, Fuyang 236041, China
}

Correspondence should be addressed to Wei Kang; kangwei0830@163.com

Received 30 November 2014; Accepted 24 December 2014

Academic Editor: Yun-Bo Zhao

Copyright (C) 2015 Wei Kang et al. This is an open access article distributed under the Creative Commons Attribution License, which permits unrestricted use, distribution, and reproduction in any medium, provided the original work is properly cited.

\begin{abstract}
The problem of passivity analysis for discrete-time stochastic neural networks with time-varying delays is investigated in this paper. New delay-dependent passivity conditions are obtained in terms of linear matrix inequalities. Less conservative conditions are obtained by using integral inequalities to aid in the achievement of criteria ensuring the positiveness of the Lyapunov-Krasovskii functional. At last, numerical examples are given to show the effectiveness of the proposed method.
\end{abstract}

\section{Introduction}

Neural networks have been greatly applied in many areas in the past few decades, such as static processing, pattern recognition, and combinatorial optimization [1-3]. In practice, time-delays are frequently encountered in neural networks. As the finite signal propagation time and the finite speed of information processing, the existence of the delays may cause oscillation, instability, and divergence in neural networks. Moreover, stochastic perturbations and parameter uncertainties are two main resources which could reduce the performances of delayed neural networks. Due to the importance in both theory and practice, the problem of stability for stochastic delayed neural networks with parameter uncertainties is one of hot issues. Therefore, there have been lots of important and interesting results in this field [3-17].

It should be noticed that most neural networks are focused on continuous-time case [3, 7-12]. However, discrete-time systems play crucial roles in today's information society. Particulary, when implementing the delayed continuous-time neural networks for computer simulation, it needs to formulate discrete-time system. Thus, it is necessary to research the dynamics of discrete-time neural networks. In recent years, a lot of important results have been published in the literatures [13-17]. Kwon et al. [14] discussed the stability criteria for the discrete-time system with time varying delays. Wang et al. [16] researched the exponential stability of discrete-time neural networks with distributed delays by means of Lyapunov-Krasovskill functional theory and linear matrix inequalities technology. In [17], the authors are concerned with the robust state estimation for discrete neural networks with successive packet dropouts, linear fractional uncertainties, and mixed time-delays.

On the other hand, passivity is a significant concept that represents input-output feature of dynamic systems, which can offer a powerful tool for analyzing mechanical systems, nonlinear systems, and electrical circuits [18]. The passivity theory was firstly presented in the circuit analysis [19]. During the past several decades, the passivity theory has found successful applications in various areas such as complexity, signal processing, stability, chaos control, and fuzzy control. Thus, the problem of passivity for time-delay neural networks has received much attention and lots of effective approaches have been proposed in this research area [20-27]. The authors $[21,22]$ discussed the problem of passivity for neural networks with time-delays. Recently, Lee et al. [23] further studied the problem of dissipative analysis for neural networks with times-delays by using reciprocally convex approach and 
linear matrix inequality technology. Very recently, in [24], the problem of passivity criterion of discrete-time stochastic bidirectional associative memory neural networks with timevarying delays has been developed. In [25], some delaydependent sufficient passivity conditions have been obtained for stochastic discrete-time neural networks with timevarying delays in terms of linear matrix inequalities technology and free-weighting matrices approach. A less conservative passivity criterion for discrete-time stochastic neural networks with time-varying delays was derived in [26]. However, there is still a room for decreasing the conservatism.

Motivated by the above discussion, the problem of passivity for discrete-time stochastic neural networks with timevarying delays is studied. The major contribution of this paper lies in that, first of all, different from the traditional way, a new inequality is introduced to deal with terms $\sum_{i=k-\tau_{m}}^{k-1} \eta^{T}(i) T_{1} \eta(i)$ and $\sum_{i=k-\tau_{M}}^{k-\tau_{m}-1} \eta^{T}(i) T_{1} \eta(i)$. This method can effectively reduce the conservatism. Secondly, we do not need all the symmetric matrices in the Lyapunov functional to be positive definite and take advantage of the relationships of $\tau(k)-\tau_{m}$ and $\tau_{M}-\tau(k)$. New passivity conditions are presented in terms of matrix inequalities. Finally, numerical examples are given to indicate the effectiveness of the proposed method.

Notations. Throughout this paper, the superscripts " -1 " and " $T$ " stand for the inverse and transpose of a matrix, respectively; $P>0(P \geqslant 0, P<0, P \leqslant 0)$ means that the matrix $P$ is symmetric positive definite (positive semidefinite, negative definite, and negative semidefinite); $E\{\cdot\}$ stands for the mathematical expectation operator with respect to the given probability measure; $\|\cdot\|$ refers to the Euclidean vector norm; $(\Omega, \mathscr{F}, \mathscr{P})$ denotes a complete probability space with a filtration containing all $p$-null sets and is right conditions; $N[a, b]$ denotes the discrete interval given $N[a, b]=\{a, a+1, \ldots, b-$ $1, b\} ; R^{n}$ denotes $n$-dimensional Euclidean space; $R^{m \times n}$ is the set of $m \times n$ real matrices; $*$ denotes the symmetric block in symmetric matrix; $\lambda_{\max }(Q)$ and $\lambda_{\min }(Q)$ denote, respectively, the maximal and minimal eigenvalue of matrix $Q$.

\section{Problem Statement and Preliminaries}

Consider the following DSNN with time-varying delays:

$$
\begin{aligned}
x(k+1)= & A x(k)+B_{1} g(x(k))+B_{2} g(x(k-\tau(k))) \\
& +u(k)+\delta(x(k), x(k-\tau(k))) \omega(k), \\
y(k)= & g(x(k)), \\
x(j)= & \psi(j), \quad j=-\tau_{M},-\tau_{M}+1, \ldots, 0,
\end{aligned}
$$

where $x(k)=\left[x_{1}(k), x_{2}(k), \ldots, x_{n}(k)\right]^{T} \in R^{n}$ is the neuron state vector of the system; $y(k)$ is the output of the neural networks; $u(k)=\left[u_{1}(k), u_{2}(k), \ldots, u_{n}(k)\right]^{T}$ is the input vector; $\psi(j)$ is the initial condition; $A=\operatorname{diag}\left(a_{1}, a_{2}, \ldots, a_{n}\right)$ is the state feedback coefficient matrix; $B_{1}$ and $B_{2}$ are the connection weight matrices and the delayed connection weight matrices, respectively; $g(x(k))=\left[g_{1}\left(x_{1}(k)\right)\right.$, $\left.g_{2}\left(x_{2}(k)\right), \ldots, g_{n}\left(x_{n}(k)\right)\right]^{T} \in R^{n}$ represents the neuron activation functions; $\tau(k)$ denotes the known time-varying delay and satisfies $0<\tau_{m} \leq \tau(k) \leq \tau_{M}$; $\delta$ is diffusion coefficient vector and $\omega(k)$ is a scalar Brownian motion defined on the probability space $(\Omega, \mathscr{F}, \mathscr{P})$ with

$$
\begin{aligned}
E\{\omega(k)\} & =0, \\
E\{\omega(k) \omega(k)\} & =1, \\
E\{\omega(i) \omega(j)\} & =0, \quad(i \neq j) .
\end{aligned}
$$

Assumption 1. The neuron activation function $g(\cdot)$ satisfies

$$
l_{s}^{-} \leqslant \frac{g_{s}(a)-g_{s}(b)}{a-b} \leqslant l_{s}^{+}, \quad g_{s}(0)=0, s=1,2, \ldots, n,
$$

for all $a, b \in R, a \neq b$, where $l_{s}^{-}$and $l_{s}^{+}$are known real constants.

Remark 2. In Assumption $1, l_{s}^{-}$and $l_{s}^{+}$can be positive, negative, or zero. Moreover, when $l_{s}^{-}=0$, then $l_{s}^{+}>0$.

Assumption 3. $\delta(x(k), x(k-\tau(k)))$ is the continuous function satisfying

$$
\begin{aligned}
& \delta^{T}(x(k), x(k-\tau(k))) \delta(x(k), x(k-\tau(k))) \\
& \quad \leq \rho_{1} x^{T}(k) x(k)+\rho_{2} x^{T}(k-\tau(k)) x(k-\tau(k)),
\end{aligned}
$$

where $\rho_{1}, \rho_{2}$ are known constant scalars.

The following lemmas and definition will be used in proof of main results.

Lemma 4. For integers $\tau(k)$ and vector function $x(k+\cdot)$ : $N\left[-\tau_{M},-\tau_{m}\right] \mapsto R^{n}, \eta(k)=x(k+1)-x(k)$, for any positive semidefinite matrix

$$
X=\left[\begin{array}{ccc}
X_{11} & X_{12} & X_{13} \\
X_{12}^{T} & X_{22} & X_{23} \\
X_{13}^{T} & X_{23}^{T} & X_{33}
\end{array}\right] \geq 0,
$$

the following inequality holds:

$$
\begin{gathered}
-\sum_{i=k-\tau(k)}^{k-1} \eta^{T}(i) X_{33} \eta(i) \\
\leq \sum_{i=k-\tau(k)}^{k-1}\left[\begin{array}{ll}
x^{T}(k) & x^{T}(k-\tau(k)) \eta^{T}(i)
\end{array}\right] \\
\cdot\left[\begin{array}{ccc}
X_{11} & X_{12} & X_{13} \\
X_{12}^{T} & X_{22} & X_{23} \\
X_{13}^{T} & X_{23}^{T} & 0
\end{array}\right]\left[\begin{array}{c}
x(k) \\
x(k-\tau(k)) \\
\eta(i)
\end{array}\right] .
\end{gathered}
$$


Proof. In fact, we have

$$
\begin{aligned}
& 0 \leq \sum_{i=k-\tau(k)}^{k-1}\left[x^{T}(k) x^{T}(k-\tau(k)) \eta^{T}(i)\right] \\
& \cdot\left[\begin{array}{ccc}
X_{11} & X_{12} & X_{13} \\
X_{12}^{T} & X_{22} & X_{23} \\
X_{13}^{T} & X_{23}^{T} & X_{33}
\end{array}\right]\left[\begin{array}{c}
x(k) \\
x(k-\tau(k)) \\
\eta(i)
\end{array}\right] \\
& =\sum_{i=k-\tau(k)}^{k-1}\left(\eta^{T}(i) X_{33} \eta(i)\right. \\
& +\left[\begin{array}{lll}
x^{T}(k) & x^{T}(k-\tau(k)) & \eta^{T}(i)
\end{array}\right] \\
& \left.\cdot\left[\begin{array}{ccc}
X_{11} & X_{12} & X_{13} \\
X_{12}^{T} & X_{22} & X_{23} \\
X_{13}^{T} & X_{23}^{T} & 0
\end{array}\right]\left[\begin{array}{c}
x(k) \\
x(k-\tau(k)) \\
\eta(i)
\end{array}\right]\right) .
\end{aligned}
$$

Thus, one can easily obtain

$$
\begin{aligned}
& -\sum_{i=k-\tau(k)}^{k-1} \eta^{T}(i) X_{33} \eta(i) \\
& \leq \sum_{i=k-\tau(k)}^{k-1}\left[x^{T}(k) x^{T}(k-\tau(k)) \eta^{T}(i)\right] \\
& \cdot\left[\begin{array}{ccc}
X_{11} & X_{12} & X_{13} \\
X_{12}^{T} & X_{22} & X_{23} \\
X_{13}^{T} & X_{23}^{T} & 0
\end{array}\right]\left[\begin{array}{c}
x(k) \\
x(k-\tau(k)) \\
\eta(i)
\end{array}\right] .
\end{aligned}
$$

The proof is completed.

Remark 5. The new inequality was proposed in $[5,6]$ for continuous-time systems; it is worth noting that we firstly extend this method to study discrete-time neural networks in this paper.

Lemma 6 (see $[4,13]$ ). Let $M \in R^{n \times n}$ be a positive-definite matrix, $X_{i} \in R^{n}$; then

$$
\begin{aligned}
& -(m-n) \sum_{i=k-m}^{k-n-1} X_{i}^{T} M X_{i} \\
& \leqslant-\left(\sum_{i=k-m}^{k-n-1} X_{i}\right)^{T} M\left(\sum_{i=k-m}^{k-n-1} X_{i}\right) .
\end{aligned}
$$

Lemma 7 (see [24]). Let $A=A^{T}, D, E$ be real matrices with appropriate dimensions, with matrix $F(k)$ satisfying $F^{T}(k) F(k) \leq I$; there exists a scalar $\varepsilon>0$ such that

$$
A+D F(t) E+E^{T} F^{T}(t) D^{T} \leq A+\varepsilon D D^{T}+\varepsilon^{-1} E^{T} E .
$$

Lemma 8 (see [3]). For any constant matrices $\Omega, \Upsilon_{1}, \Upsilon_{2}$ with appropriate dimensions, and a function $\tau(k)$ satisfying $\tau_{m} \leq$ $\tau(k) \leq \tau_{M}$, then

$$
\Omega+\left(\tau(k)-\tau_{m}\right) \Upsilon_{1}+\left(\tau_{M}-\tau(k)\right) \Upsilon_{2}<0,
$$

if and only if

$$
\begin{aligned}
& \Omega+\left(\tau_{M}-\tau_{m}\right) \Upsilon_{1}<0, \\
& \Omega+\left(\tau_{M}-\tau_{m}\right) \Upsilon_{2}<0 .
\end{aligned}
$$

Definition 9 (see [25]). The system (1) is said to be passive if there exists a scalar $\gamma>0$ satisfying

$$
2 \sum_{i=0}^{k_{0}} E\left\{y^{T}(i) u(i)\right\} \geq-\gamma \sum_{i=0}^{k_{0}} E\left\{u^{T}(i) u(i)\right\},
$$

for all $k_{0} \in N$ and for all solution of (1) with $\psi(0)=0$.

\section{Main Results}

In this section, the passivity of discrete-time stochastic neural networks with time-varying delays will be investigated by use of the new integral inequality and Lyapunov method. In the paper, some of symmetric matrices in Lyapunov-Krasovskii functional are not necessarily required to be positive definite.

Denote

$$
\begin{aligned}
L_{1} & =\operatorname{diag}\left\{l_{1}^{-} l_{1}^{+}, \ldots, l_{n}^{-} l_{n}^{+}\right\}, \\
L_{2} & =\operatorname{diag}\left\{\frac{l_{1}^{-}+l_{1}^{+}}{2}, \ldots, \frac{l_{n}^{-}+l_{n}^{+}}{2}\right\}, \\
\tau_{M m} & =\tau_{M}-\tau_{m}, \\
\eta(k) & =x(k+1)-x(k) .
\end{aligned}
$$

Main results are given in the following theorems.

Theorem 10. Under Assumptions 1 and 3, the discrete-time stochastic neural network (1) is passive, if there exist matrices $P$, $R_{1}, R_{2}, Q=\left[\begin{array}{cc}Q_{11} & Q_{12} \\ * & Q_{22}\end{array}\right]>0, T_{1}>0, T_{2}>0, X=\left[\begin{array}{ccc}X_{11} & X_{12} & X_{13} \\ * & X_{22} & X_{23} \\ * & * & X_{33}\end{array}\right] \geq$ $0, Y=\left[\begin{array}{ccc}Y_{11} & Y_{12} & Y_{13} \\ * & Y_{22} & Y_{23} \\ * & * & Y_{33}\end{array}\right] \geq 0, Z=\left[\begin{array}{ccc}Z_{11} & Z_{12} & Z_{13} \\ * & Z_{22} & Z_{23} \\ * & * & Z_{33}\end{array}\right] \geq 0$, the positive diagonal matrices $S_{k}=\operatorname{diag}\left\{s_{1 k}, s_{2 k}, \ldots, s_{n k}\right\}(k=1,2)$, and scalars $\lambda>0, \gamma>0$, such that the following matrix inequalities hold:

$$
\begin{gathered}
{\left[\begin{array}{cc}
T_{1}+\frac{\tau_{m}}{\tau_{M}} P & -T_{1} \\
* & T_{1}+\tau_{m} R_{1}
\end{array}\right]>0,} \\
{\left[\begin{array}{cc}
T_{2}+P & -T_{2} \\
* & T_{2}+\tau_{M} R_{2}
\end{array}\right]>0,}
\end{gathered}
$$

$$
\begin{gathered}
T_{1}-X_{33} \geq 0, \\
T_{2}-Y_{33} \geq 0, \\
T_{2}-Z_{33} \geq 0, \\
\Theta \leq \lambda I, \\
\Omega+\Upsilon_{1}<0, \\
\Omega+\Upsilon_{2}<0,
\end{gathered}
$$


where

$$
\begin{aligned}
& \Theta=P+\tau_{m} T_{1}+\tau_{M m} T_{2}, \\
& \Omega=\left[\begin{array}{ccc}
\Omega_{11} & \cdots & \Omega_{17} \\
* & \ddots & \vdots \\
* & * & \Omega_{77}
\end{array}\right] \text {, } \\
& \Upsilon_{1}=\left[\begin{array}{ccc}
d_{11} & \cdots & d_{17} \\
* & \ddots & \vdots \\
* & * & d_{77}
\end{array}\right] \text {, } \\
& \Upsilon_{2}=\left[\begin{array}{ccc}
e_{11} & \cdots & e_{17} \\
* & \ddots & \vdots \\
* & * & e_{77}
\end{array}\right] \text {, } \\
& \Omega_{11}=\left(\tau_{M m}+1\right) Q_{11}+R_{1}+\tau_{m} X_{11}+X_{13}+X_{13}^{T} \\
& +\tau_{m}(A-I) T_{1}(A-I)+\tau_{M m}(A-I) T_{2}(A-I) \\
& +\rho_{1} \lambda I+A P A-P-S_{1} L_{1} \\
& \Omega_{12}=\tau_{m} X_{12}-X_{13}+X_{23}^{T} \text {, } \\
& \Omega_{15}=\left(\tau_{M m}+1\right) Q_{12}+L_{2} S_{1}+\tau_{m}(A-I) T_{1} B_{1} \\
& +\tau_{M m}(A-I) T_{2} B_{1}+A^{T} P B_{1}, \\
& \Omega_{16}=\tau_{m}(A-I) T_{1} B_{1}+\tau_{M m}(A-I) T_{2} B_{1}+A P B_{1} \text {, } \\
& \Omega_{17}=\tau_{m}(A-I) T_{1}+\tau_{M m}(A-I) T_{2}+A P, \\
& \Omega_{22}=-R_{1}+R_{2}+\tau_{m} X_{22}-X_{23}-X_{23}^{T}+Y_{13}+Y_{13}^{T} \text {, } \\
& \Omega_{23}=-Y_{13}+Y_{23}^{T} \text {, } \\
& \Omega_{33}=-Q_{11}-S_{2} L_{1}+\rho_{2} \lambda I-Y_{23}-Y_{23}^{T}+Z_{13}+Z_{13}^{T} \text {, } \\
& \Omega_{34}=-Z_{13}+Z_{23}^{T} \text {, } \\
& \Omega_{36}=-Q_{12}+L_{2} S_{2} \text {, } \\
& \Omega_{44}=-R_{2}-Z_{23}-Z_{23}^{T} \text {, } \\
& \Omega_{55}=B_{1}^{T} P B_{1}+\tau_{m} B_{1}^{T} T_{1} B_{1}+\tau_{M m} B_{1}^{T} T_{2} B_{1} \\
& +\left(\tau_{M m}+1\right) Q_{22}-S_{1} \text {, } \\
& \Omega_{56}=B_{1}^{T} P B_{2}+\tau_{m} B_{1}^{T} T_{1} B_{2}+\tau_{M m} B_{1}^{T} T_{2} B_{2}, \\
& \Omega_{57}=B_{1}^{T} P+\tau_{m} B_{1}^{T} T_{1}+\tau_{M m} B_{1}^{T} T_{2}-I, \\
& \Omega_{66}=B_{2}^{T} P B_{2}+\tau_{m} B_{2}^{T} T_{1} B_{2}+\tau_{M m} B_{2}^{T} T_{2} B_{2} \\
& -Q_{22}-S_{2} \\
& \Omega_{67}=B_{2}^{T} P+\tau_{m} B_{2}^{T} T_{1}+\tau_{M m} B_{2}^{T} T_{2}, \\
& \Omega_{77}=P+\tau_{m} T_{1}+\tau_{M m} T_{2}-\gamma I, \\
& d_{22}=Y_{11} \text {, }
\end{aligned}
$$

$$
\begin{aligned}
& d_{23}=Y_{12}, \\
& d_{33}=Y_{22}, \\
& e_{33}=Z_{11}, \\
& e_{34}=Y_{12}, \\
& d_{44}=Z_{22}, \quad \text { elsewhere, } \\
& \Omega_{i j}=0, \\
& d_{i j}=0, \\
& e_{i j}=0, \\
& i, j=1,2, \ldots, 8 .
\end{aligned}
$$

Proof. Define a new augmented of Lyapunov-Krasovskii functional as follows:

$$
V(k)=V_{1}(k)+V_{2}(k)+V_{3}(k)+V_{4}(k)
$$

where

$$
\begin{aligned}
V_{1}(k)= & x^{T}(k) P x(k) \\
V_{2}(k)= & \sum_{i=k-\tau_{m}}^{k-1} x^{T}(i) R_{1} x(i)+\sum_{i=k-\tau_{M}}^{k-\tau_{m}-1} x^{T}(i) R_{2} x(i) \\
V_{3}(k)= & \sum_{i=k-\tau(k)}^{k-1}\left[\begin{array}{c}
x(i) \\
g(x(i))
\end{array}\right]^{T}\left[\begin{array}{cc}
Q_{11} & Q_{12} \\
* & Q_{22}
\end{array}\right]\left[\begin{array}{c}
x(i) \\
g(x(i))
\end{array}\right] \\
& +\sum_{j=-\tau_{M}}^{-\tau_{m}-1} \sum_{i=k+j}^{k-1}\left[\begin{array}{c}
x(i) \\
g(x(i))
\end{array}\right]^{T}\left[\begin{array}{cc}
Q_{11} & Q_{12} \\
* & Q_{22}
\end{array}\right]\left[\begin{array}{c}
x(i) \\
g(x(i))
\end{array}\right], \\
V_{4}(k)= & \sum_{j=-\tau_{m}}^{-1} \sum_{i=k+j}^{k-1} \eta^{T}(i) T_{1} \eta(i) \\
& +\sum_{j=-\tau_{M}-1} \sum_{i=k+j}^{k-1} \eta^{T}(i) T_{2} \eta(i)
\end{aligned}
$$

Firstly, we show that the Lyapunov-Krasovskii functional $V(k)$ is positive definite. By using Lemma 6, one can obtain

$$
\begin{aligned}
& \sum_{j=-\tau_{m}}^{-1} \sum_{i=k+j}^{k-1} \eta^{T}(i) T_{1} \eta(i) \\
& \geq \sum_{j=-\tau_{m}}^{-1} \frac{-1}{j}\left(\sum_{i=k+j}^{k-1} \eta(i)\right)^{T} T_{1}\left(\sum_{i=k+j}^{k-1} \eta(i)\right) \\
& =\sum_{j=-\tau_{m}}^{-1} \frac{-1}{j}\left[\begin{array}{c}
x(k) \\
x(k+j)
\end{array}\right]^{T}\left[\begin{array}{cc}
T_{1} & -T_{1} \\
* & T_{1}
\end{array}\right]\left[\begin{array}{c}
x(k) \\
x(k+j)
\end{array}\right] \\
& \geq \frac{1}{\tau_{m}} \sum_{i=k-\tau_{m}}^{k-1}\left[\begin{array}{c}
x(k) \\
x(i)
\end{array}\right]^{T}\left[\begin{array}{cc}
T_{1} & -T_{1} \\
* & T_{1}
\end{array}\right]\left[\begin{array}{c}
x(k) \\
x(i)
\end{array}\right],
\end{aligned}
$$




$$
\begin{aligned}
& \sum_{j=-\tau_{M}}^{-\tau_{m}-1} \sum_{i=k+j}^{k-1} \eta^{T}(i) T_{2} \eta(i) \\
& \geq \sum_{j=-\tau_{M}}^{-\tau_{m}-1} \frac{-1}{j}\left(\sum_{i=k+j}^{k-1} \eta(i)\right)^{T} Z_{2}\left(\sum_{i=k+j}^{k-1} \eta(i)\right) \\
& =\sum_{j=-\tau_{M}}^{-\tau_{m}-1} \frac{-1}{j}\left[\begin{array}{c}
x(k) \\
x(k+j)
\end{array}\right]^{T}\left[\begin{array}{cc}
T_{1} & -T_{1} \\
* & T_{1}
\end{array}\right]\left[\begin{array}{c}
x(k) \\
x(k+j)
\end{array}\right] \\
& \geq \frac{1}{\tau_{M}} \sum_{i=k-\tau_{M}}^{k-\tau_{m}-1}\left[\begin{array}{c}
x(k) \\
x(i)
\end{array}\right]^{T}\left[\begin{array}{cc}
T_{2} & -T_{2} \\
* & T_{2}
\end{array}\right]\left[\begin{array}{c}
x(k) \\
x(i)
\end{array}\right],
\end{aligned}
$$

$$
\begin{aligned}
x^{T}(k) & P x(k) \\
= & \sum_{i=k-\tau_{m}}^{k-1} \frac{1}{\tau_{M}} x^{T}(k) P x(k) \\
& +\sum_{i=k-\tau_{M}}^{k-\tau_{m}-1} \frac{1}{\tau_{M}} x^{T}(k) P x(k) .
\end{aligned}
$$

Then, it follows from (19)-(21) that

$$
\begin{aligned}
V(k) \geq & \sum_{i=k-\tau_{m}}^{k-1} \frac{1}{\tau_{M}} x^{T}(k) P x(k)+\sum_{i=k-\tau_{M}}^{k-\tau_{m}-1} \frac{1}{\tau_{M}} x^{T}(k) P x(k) \\
& +\sum_{i=k-\tau_{m}}^{k-1} x^{T}(i) R_{1} x(i)+\sum_{i=k-\tau_{M}}^{k-\tau_{m}-1} x^{T}(i) R_{2} x(i) \\
& +\frac{1}{\tau_{m}} \sum_{i=k-\tau_{m}}^{k-1}\left[\begin{array}{c}
x(k) \\
x(i)
\end{array}\right]^{T}\left[\begin{array}{cc}
T_{1} & -T_{1} \\
* & T_{1}
\end{array}\right]\left[\begin{array}{c}
x(k) \\
x(i)
\end{array}\right] \\
& +\frac{1}{\tau_{M}} \sum_{i=k-\tau_{M}}^{k-\tau_{m}-1}\left[\begin{array}{c}
x(k) \\
x(i)
\end{array}\right]^{T}\left[\begin{array}{cc}
T_{2} & -T_{2} \\
* & T_{2}
\end{array}\right]\left[\begin{array}{c}
x(k) \\
x(i)
\end{array}\right] \\
\geq & \frac{1}{\tau_{m}} \sum_{i=k-\tau_{m}}^{k-1}\left[\begin{array}{c}
x(k) \\
x(i)
\end{array}\right]^{T}\left[\begin{array}{cc}
T_{1}+\frac{\tau_{m}}{\tau_{M}} P & -T_{1} \\
-T_{1} & T_{1}+\tau_{m} R_{1}
\end{array}\right]\left[\begin{array}{c}
x(k) \\
x(i)
\end{array}\right] \\
& +\frac{1}{\tau_{M}} \sum_{i=k-\tau_{M}}^{k-\tau_{m}-1}\left[\begin{array}{c}
x(k) \\
x(i)
\end{array}\right]^{T}\left[\begin{array}{cc}
T_{2}+P & -T_{2} \\
-T_{2} & T_{2}+\tau_{m} R_{2}
\end{array}\right]\left[\begin{array}{c}
x(k) \\
x(i)
\end{array}\right] .
\end{aligned}
$$

From condition (15), there exists a scalar $\delta_{1}>0$, for any $x(k) \neq 0$, such that

$$
V(k) \geq \delta_{1}\|x(k)\|^{2}>0 .
$$

Now, taking the forward difference of $V(k)$ along the trajectories of system (1), it yields that

$$
\begin{aligned}
& E\left\{\Delta V_{1}(k)\right\} \\
& =E\left\{(\eta(k)+x(k))^{T} P(\eta(k)+x(k))-x^{T}(k) P x(k)\right\} \\
& =E\left\{\eta^{T}(k) P \eta(k)+2 x^{T}(k) P \eta(k)\right\}, \\
& E\left\{\Delta V_{2}(k)\right\} \\
& =E\left\{x^{T}(k) R_{1} x(k)-x^{T}\left(k-\tau_{m}\right)\left(R_{1}-R_{2}\right) x\left(k-\tau_{m}\right)\right. \\
& \left.-x^{T}\left(k-\tau_{M}\right) R_{2} x\left(k-\tau_{M}\right)\right\}, \\
& \leq E\left\{\left(\tau_{M m}+1\right)\left[\begin{array}{c}
x(k) \\
g(x(k))
\end{array}\right]^{T}\left[\begin{array}{cc}
Q_{11} & Q_{12} \\
* & Q_{22}
\end{array}\right]\left[\begin{array}{c}
x(k) \\
g(x(k))
\end{array}\right]\right. \\
& -\left[\begin{array}{c}
x(k-\tau(k)) \\
g(x(k-\tau(k)))
\end{array}\right]^{T}\left[\begin{array}{cc}
Q_{11} & Q_{12} \\
* & Q_{22}
\end{array}\right] \\
& \left.\cdot\left[\begin{array}{c}
x(k-\tau(k)) \\
g(x(k-\tau(k)))
\end{array}\right]\right\} \\
& =E\left\{\left(\tau_{M m}+1\right) x^{T}(k) Q_{11} x(k)+2 x^{T}(k) Q_{12} g(x(k))\right. \\
& +g^{T}(x(k)) Q_{22} g(x(k)) \\
& \text { - } x^{T}(k-\tau(k)) Q_{11} x(k-\tau(k)) \\
& -2 x^{T}(k-\tau(k)) Q_{12} g(x(k-\tau(k))) \\
& \left.-g^{T}(x(k-\tau(k))) Q_{22} g(x(k-\tau(k)))\right\},
\end{aligned}
$$

$E\left\{\Delta V_{4}(k)\right\}$

$$
\begin{gathered}
=E\left\{\eta^{T}(k)\left(\tau_{m} T_{1}+\tau_{M m} T_{2}\right) \eta(k)-\sum_{i=k-\tau_{m}}^{k-1} \eta^{T}(i) T_{1} \eta(i)\right. \\
\left.\quad-\sum_{i=k-\tau(k)}^{k-\tau_{m}-1} \eta^{T}(i) T_{2} \eta(i)-\sum_{i=k-\tau_{M}}^{k-\tau(k)-1} \eta^{T}(i) T_{2} \eta(i)\right\},
\end{gathered}
$$$$
E\left\{-\sum_{i=k-\tau_{m}}^{k-1} \eta^{T}(i) T_{1} \eta(i)-\sum_{i=k-\tau(k)}^{k-\tau_{m}-1} \eta^{T}(i) T_{2} \eta(i)\right.
$$$$
\left.-\sum_{i=k-\tau_{M}}^{k-\tau(k)-1} \eta^{T}(i) T_{2} \eta(i)\right\}
$$$$
=E\left\{-\sum_{i=k-\tau_{m}}^{k-1} \eta^{T}(i)\left(T_{1}-X_{33}\right) \eta(i)\right.
$$$$
-\sum_{i=k-\tau(k)}^{k-\tau_{m}-1} \eta^{T}(i)\left(T_{2}-Y_{33}\right) \eta(i)
$$ 


$$
\begin{aligned}
& -\sum_{i=k-\tau_{M}}^{k-\tau(k)-1} \eta^{T}(i)\left(T_{2}-Z_{33}\right) \eta(i)-\sum_{i=k-\tau_{m}}^{k-1} \eta^{T}(i) X_{33} \eta(i) \\
& \left.-\sum_{i=k-\tau(k)}^{k-\tau_{m}-1} \eta^{T}(i) Y_{33} \eta(i)-\sum_{i=k-\tau_{M}}^{k-\tau(k)-1} \eta^{T}(i) Z_{33} \eta(i)\right\} .
\end{aligned}
$$

Form the new inequality of Lemma 4 , one can get

$$
\begin{aligned}
& -\sum_{i=k-\tau_{m}}^{k-1} \eta^{T}(i) X_{33} \eta(i) \\
& \leq \sum_{i=k-\tau_{m}}^{k-1}\left[x^{T}(k) x^{T}\left(k-\tau_{m}\right) \eta^{T}(i)\right] \\
& \cdot\left[\begin{array}{ccc}
X_{11} & X_{12} & X_{13} \\
X_{12}^{T} & X_{22} & X_{23} \\
X_{13}^{T} & X_{23}^{T} & 0
\end{array}\right]\left[\begin{array}{c}
x(k) \\
x\left(k-\tau_{m}\right) \\
\eta(i)
\end{array}\right] \\
& =x^{T}(k)\left(\tau_{m} X_{11}+X_{13}^{T}+X_{13}\right) x(k) \\
& +x^{T}(k)\left(\tau_{m} X_{12}-X_{13}+X_{23}^{T}\right) x\left(k-\tau_{m}\right) \\
& +x^{T}\left(k-\tau_{m}\right)\left(\tau_{m} X_{12}^{T}-X_{13}^{T}+X_{23}\right) x(k) \\
& +x^{T}\left(k-\tau_{m}\right)\left(\tau_{m} X_{22}-X_{23}^{T}-X_{23}\right) x\left(k-\tau_{m}\right), \\
& -\sum_{i=k-\tau(k)}^{k-\tau_{m}-1} \eta^{T}(i) Y_{33} \eta(i) \\
& \leq \sum_{i=k-\tau(k)}^{k-\tau_{m}-1}\left[x^{T}\left(k-\tau_{m}\right) x^{T}(k-\tau(k)) \eta^{T}(i)\right] \\
& \cdot\left[\begin{array}{ccc}
X_{11} & X_{12} & X_{13} \\
X_{12}^{T} & X_{22} & X_{23} \\
X_{13}^{T} & X_{23}^{T} & 0
\end{array}\right]\left[\begin{array}{c}
x\left(k-\tau_{m}\right) \\
x(k-\tau(k)) \\
\eta(i)
\end{array}\right] \\
& =x^{T}\left(k-\tau_{m}\right)\left(\left(\tau(k)-\tau_{m}\right) Y_{11}+Y_{13}^{T}+Y_{13}\right) x\left(k-\tau_{m}\right) \\
& +x^{T}\left(k-\tau_{m}\right)\left(\left(\tau(k)-\tau_{m}\right) Y_{12}-Y_{13}+Y_{23}^{T}\right) x(k-\tau(k)) \\
& +x^{T}(k-\tau(k))\left(\left(\tau(k)-\tau_{m}\right) Y_{12}^{T}-Y_{13}^{T}+Y_{23}\right) x\left(k-\tau_{m}\right) \\
& +x^{T}(k-\tau(k))\left(\left(\tau(k)-\tau_{m}\right) Y_{22}-Y_{23}^{T}-Y_{23}\right) x(k-\tau(k)), \\
& -\sum_{i=k-\tau_{M}}^{k-\tau(k)-1} \eta^{T}(i) Z_{33} \eta(i) \\
& \leq \sum_{i=k-\tau_{M}}^{k-\tau(k)-1}\left[x^{T}(k-\tau(k)) x^{T}\left(k-\tau_{M}\right) \eta^{T}(i)\right] \\
& \cdot\left[\begin{array}{ccc}
X_{11} & X_{12} & X_{13} \\
X_{12}^{T} & X_{22} & X_{23} \\
X_{13}^{T} & X_{23}^{T} & 0
\end{array}\right]\left[\begin{array}{c}
x(k-\tau(k)) \\
x\left(k-\tau_{M}\right) \\
\eta(i)
\end{array}\right]
\end{aligned}
$$

$$
\begin{aligned}
= & x^{T}(k-\tau(k))\left(\left(\tau_{M}-\tau(k)\right) Z_{11}+Z_{13}^{T}+Z_{13}\right) x(k-\tau(k)) \\
& +x^{T}(k-\tau(k))\left(\left(\tau_{M}-\tau(k)\right) Z_{12}-Z_{13}+Z_{23}^{T}\right) x\left(k-\tau_{M}\right) \\
& +x^{T}\left(k-\tau_{M}\right)\left(\left(\tau_{M}-\tau(k)\right) Z_{12}^{T}-Z_{13}^{T}+Z_{23}\right) x(k-\tau(k)) \\
& +x^{T}\left(k-\tau_{M}\right)\left(\left(\tau_{M}-\tau(k)\right) Z_{22}-Z_{23}^{T}-Z_{23}\right) x\left(k-\tau_{M}\right) .
\end{aligned}
$$

It is easy to get

$$
\begin{aligned}
& E\left\{\eta^{T}(k) \Theta \eta(k)\right\} \\
& =E\left\{\left[(A-I) x(k)+B_{1} g(x(k))+B_{2} g(x(k-\tau(k)))\right.\right. \\
& +u(k)+\delta(x(k), x(k-\tau(k))) \omega(k)]^{T} \\
& \cdot \Theta\left[(A-I) x(k)+B_{1} g(x(k))\right. \\
& \quad+B_{2} g(x(k-\tau(k))) \\
& +u(k)+\delta(x(k), x(k-\tau(k))) \omega(k)]\} .
\end{aligned}
$$

From Assumption 3 and inequality (16), we have

$$
\begin{aligned}
& \delta(x(k), x(k-\tau(k))) \Theta \delta(x(k), x(k-\tau(k))) \\
& \quad \leq \lambda\left[\rho_{1} x^{T}(k) x(k)+\rho_{2} x^{T}(k-\tau(k)) x(k-\tau(k))\right] .
\end{aligned}
$$

From Assumption 1, it follows that

$$
\begin{aligned}
& \left(g_{i}\left(x_{i}(k)\right)-l_{i}^{+} x_{i}(k)\right)\left(g_{i}\left(x_{i}(k)\right)-l_{i}^{-} x_{i}(k)\right) \leq 0, \\
& \left(g_{i}\left(x_{i}(k-\tau(k))\right)-l_{i}^{+} x_{i}(k-\tau(k))\right) \\
& \quad \cdot\left(g_{i}\left(x_{i}(k-\tau(k))\right)-l_{i}^{-} x_{i}(k-\tau(k))\right) \leq 0 .
\end{aligned}
$$

Thus, for the diagonal matrices $S_{k}=\operatorname{diag}\left\{s_{1 k}, s_{2 k}, \ldots, s_{n k}\right\}$ $(k=1,2)$, one can receive the following inequalities:

$$
\begin{gathered}
-\left[\begin{array}{c}
x(k) \\
g(x(k))
\end{array}\right]^{T}\left[\begin{array}{cc}
S_{1} L_{1} & -S_{1} L_{2} \\
* & S_{1}
\end{array}\right]\left[\begin{array}{c}
x(k) \\
g(x(k))
\end{array}\right] \geq 0, \\
-\left[\begin{array}{c}
x(k-\tau(k)) \\
g(x(k-\tau(k)))
\end{array}\right]^{T} \\
\cdot\left[\begin{array}{cc}
S_{2} L_{1} & -S_{2} L_{2} \\
* & S_{2}
\end{array}\right]\left[\begin{array}{c}
x(k-\tau(k)) \\
g(x(k-\tau(k)))
\end{array}\right] \geq 0 .
\end{gathered}
$$


Combining (25)-(30), it yields

$$
\begin{gathered}
E\left\{\Delta V(k)-2 y^{T}(k) u(k)-\gamma u^{T}(k) u(k)\right\} \\
\leq E\left\{\alpha^{T}(k)\left(\Omega+\left(\tau(k)-\tau_{m}\right) \Upsilon_{1}+\left(\tau_{M}-\tau(k)\right) \Upsilon_{2}\right) \alpha(k)\right. \\
\quad-\sum_{i=k-\tau_{m}}^{k-1} \eta^{T}(i)\left(T_{1}-X_{33}\right) \eta(i) \\
\quad-\sum_{i=k-\tau(k)}^{k-\tau_{m}-1} \eta^{T}(i)\left(T_{2}-Y_{33}\right) \eta(i) \\
\left.-\sum_{i=k-\tau(k)-1}^{k} \eta^{T}(i)\left(T_{2}-Z_{33}\right) \eta(i)\right\},
\end{gathered}
$$

where

$$
\begin{gathered}
\alpha^{T}(k)=\left[x^{T}(k), x^{T}\left(k-\tau_{m}\right), x^{T}(k-\tau(k)), x^{T}\left(k-\tau_{M}\right),\right. \\
\left.g^{T}(x(k)), g^{T}(x(k-\tau(k))), u^{T}(k)\right] .
\end{gathered}
$$

From (15)-(17), observing that $T_{1}-X_{33} \geq 0, T_{2}-Y_{33} \geq 0$, and $T_{2}-Z_{33} \geq 0$, one can conclude that

$$
E\left\{\Delta V(k)-2 y^{T}(k) u(k)-\gamma u^{T}(k) u(k)\right\} \leq 0 .
$$

Then,

$$
\begin{aligned}
2 \sum_{i=0}^{k_{0}} E\left\{y^{T}(i) u(i)\right\} \geq & \sum_{i=0}^{k_{0}} E\{\Delta V(i)\} \\
& -\gamma \sum_{i=0}^{k_{0}} E\left\{u^{T}(i) u(i)\right\} .
\end{aligned}
$$

By the definition of $V(k)$ and inequality (23), one can find that

$$
\sum_{i=0}^{k_{0}} E\{\Delta V(i)\}=E\left\{V\left(k_{0}+1\right)-V(0)\right\} \geq 0 .
$$

So, one can have

$$
2 \sum_{i=0}^{k_{0}} E\left\{y^{T}(i) u(i)\right\} \geq-\gamma \sum_{i=0}^{k_{0}} E\left\{u^{T}(i) u(i)\right\},
$$

for all $k_{0} \in N$. This completes the proof.

Remark 11. It should be pointed out that the new inequality is introduced to deal with $\sum_{i=k-\tau_{m}}^{k-1} \eta^{T}(i) T_{1} \eta(i)$ and $\sum_{i=k-\tau_{M}}^{k-\tau_{m}-1} \eta^{T}(i) T_{1} \eta(i)$, which is immensely different from traditional ways. This method can effectively reduce the conservatism of the results.
Remark 12. In this paper, not all the matrices in the Lyapunov functional need to be positive definite. In fact, the conditions in (15) assure the positive definiteness of the Lyapunov functional; this is greatly different from traditional ways for passivity researches of discrete-time neural network, because the traditional methods always need Lyapunov matrices to be positive definite.

Remark 13. It can be seen that the term $\sum_{i=k-\tau_{M}}^{k-\tau_{m}-1} \eta^{T}(i) T_{1} \eta(i)$ is divided into two parts; the aim is to make full use of the relationship of $\tau_{M}-\tau(k)$ and $\tau(k)-\tau_{m}$, and then, taking advantage of the integral inequality and Lemma 8 , new passivity conditions are obtained in terms of LMIs.

Corollary 14. Under Assumptions 1 and 3, the discrete-time stochastic neural network (1) is passive, if there exist scalars $\lambda>$ $0, \gamma>0$, matrices $P>0, R_{1}>0, R_{2}>0, Q=\left[\begin{array}{cc}Q_{11} & Q_{12} \\ * & Q_{22}\end{array}\right]>0$, $T_{1}>0, T_{2}>0, X=\left[\begin{array}{ccc}X_{11} & X_{12} & X_{13} \\ * & X_{22} & X_{23} \\ * & * & X_{33}\end{array}\right] \geq 0, Y=\left[\begin{array}{ccc}Y_{11} & Y_{12} & Y_{13} \\ * & Y_{22} & Y_{23} \\ * & * & Y_{33}\end{array}\right] \geq$ $0, Z=\left[\begin{array}{ccc}Z_{11} & Z_{12} & Z_{13} \\ * & Z_{22} & Z_{23} \\ * & * & Z_{33}\end{array}\right] \geq 0$, and the positive diagonal matrices $S_{k}=\operatorname{diag}\left\{s_{1 k}, s_{2 k}, \ldots, s_{n k}\right\}(k=1,2)$, such that linear matrix inequalities (16) and (17) hold.

Now, we will consider the stochastic discrete-time neural networks with time-varying delay and parameter uncertainties as follows:

$$
\begin{aligned}
x(k+1)= & (A+\Delta A(k)) x(k) \\
& +\left(B_{1}+\Delta B_{1}(k)\right) g(x(k)) \\
& +\left(B_{2}+\Delta B_{2}(k)\right) g(x(k-\tau(k)))+u(k) \\
& +\delta(x(k), x(k-\tau(k))) \omega(k),
\end{aligned}
$$

where $\Delta A(k), \Delta B_{1}(k)$, and $\Delta B_{2}(k)$ denote the parameter uncertainties that are assumed to be of the form

$$
\left[\Delta A(k) \quad \Delta B_{1}(k) \quad \Delta B_{2}(k)\right]=M F(k)\left[\begin{array}{lll}
E_{1} & E_{2} & E_{3}
\end{array}\right]
$$

where $M, E_{i}(i=1,2,3)$ are known constant matrices, and $F(k)$ is the unknown matrix valued function subject to

$$
F(k)^{T} F(k) \leq I .
$$

Theorem 15. Under Assumptions 1 and 3, the discrete-time stochastic uncertain neural network (37) is robustly passive, if there exist scalars $\lambda>0, \gamma>0, \varepsilon>0$, matrices $P, R_{1}, R_{2}$, $Q=\left[\begin{array}{cc}Q_{11} & Q_{12} \\ * & Q_{22}\end{array}\right]>0, T_{1}>0, T_{2}>0, X=\left[\begin{array}{ccc}X_{11} & X_{12} & X_{13} \\ * & X_{22} & X_{23} \\ * & * & X_{33}\end{array}\right] \geq 0$, $Y=\left[\begin{array}{ccc}Y_{11} & Y_{12} & Y_{13} \\ * & Y_{22} & Y_{23} \\ * & * & Y_{33}\end{array}\right] \geq 0, Z=\left[\begin{array}{ccc}Z_{11} & Z_{12} & Z_{13} \\ * & Z_{22} & Z_{23} \\ * & * & Z_{33}\end{array}\right] \geq 0$, and the positive 
diagonal matrices $S_{k}=\operatorname{diag}\left\{s_{1 k}, s_{2 k}, \ldots, s_{n k}\right\}(k=1,2)$, such that the following matrix inequalities hold:

$$
\begin{gathered}
{\left[\begin{array}{cc}
T_{1}+\frac{\tau_{m}}{\tau_{M}} P & -T_{1} \\
* & T_{1}+\tau_{m} R_{1}
\end{array}\right]>0,} \\
{\left[\begin{array}{cc}
T_{2}+P & -T_{2} \\
* & T_{2}+\tau_{M} R_{2}
\end{array}\right]>0,} \\
T_{1}-X_{33} \geq 0, \\
T_{2}-Y_{33} \geq 0
\end{gathered}
$$

$T_{2}-Z_{33} \geq 0$,

$\Theta \leq \lambda I$,

where

$$
\begin{aligned}
& \Xi_{1}=\left[\begin{array}{lllllll}
P A & 0 & 0 & 0 & P B_{1} & P B_{2} & P
\end{array}\right]^{T}, \\
& \Xi_{2}=\left[\begin{array}{llllllll}
T_{1}(A-I) & 0 & 0 & 0 & T_{1} B_{1} & T_{1} B_{2} & T_{1}
\end{array}\right]^{T}, \\
& \Xi_{3}=\left[\begin{array}{lllllll}
T_{2}(A-I) & 0 & 0 & 0 & T_{2} B_{1} & T_{2} B_{2} & T_{2}
\end{array}\right]^{T}, \\
& \Xi_{4}=\left[\begin{array}{lllllll}
E_{1} & 0 & 0 & 0 & E_{2} & E_{3} & 0
\end{array}\right]^{T} \text {, } \\
& \Theta=P+\tau_{m} T_{1}+\tau_{M m} T_{2}, \\
& \Sigma=\left[\begin{array}{ccccccc}
\Sigma_{11} & \Sigma_{12} & 0 & 0 & \left(\tau_{M m}+1\right) Q_{12}+L_{2} S_{1} & 0 & 0 \\
* & \Sigma_{22} & -Y_{13}+Y_{23}^{T} & 0 & 0 & 0 & 0 \\
* & * & \Sigma_{33} & -Z_{13}+Z_{23}^{T} & 0 & -Q_{12}+L_{2} S_{2} & 0 \\
* & * & * & -Z_{23}-Z_{23}^{T}-R_{2} & 0 & 0 & 0 \\
* & * & * & * & \left(\tau_{M m}+1\right) Q_{22}-S_{1} & 0 & -I \\
* & * & * & * & * & -Q_{22}-S_{2} & 0 \\
* & * & * & * & * & * & \Sigma_{77}
\end{array}\right], \\
& \Sigma_{11}=\left(\tau_{M m}+1\right) Q_{11}+R_{1}+\tau_{m} X_{11}+X_{13}+X_{13}^{T}+\rho_{1} \lambda I-P-S_{1} L_{1}, \\
& \Sigma_{12}=\tau_{m} X_{12}-X_{13}+X_{23}^{T} \text {, } \\
& \Sigma_{22}=-R_{1}+R_{2}+\tau_{m} X_{22}-X_{23}-X_{23}^{T}+Y_{13}+Y_{13}^{T} \text {, } \\
& \Sigma_{33}=-Q_{11}-S_{2} L_{1}+\rho_{2} \lambda I-Y_{23}-Y_{23}^{T}+Z_{13}+Z_{13}^{T} \text {, } \\
& \Sigma_{77}=P+\tau_{m} T_{1}+\tau_{M m} T_{2}-\gamma I .
\end{aligned}
$$

\section{$\Upsilon_{1}, \Upsilon_{2}$ are the same as defined in Theorem 10.}

Proof. By replacing $A, B_{1}, B_{2}$ in (17) with $A+\Delta A(k), B_{1}+$ $\Delta B_{1}(k), B_{2}+\Delta B_{2}(k)$, respectively, then using Lemma 7 , the desired results can be obtained immediately. The proof is completed.

\section{Numerical Examples}

In this section, some numerical examples are proposed to show the effectiveness of the results obtained in this paper.
Example 1. Consider the system (1) with the following parameters:

$$
\begin{aligned}
A & =\left[\begin{array}{cc}
0.8 & 0 \\
0 & 0.9
\end{array}\right], \\
B_{1} & =\left[\begin{array}{cc}
0.001 & 0 \\
0 & 0.005
\end{array}\right], \\
B_{2} & =\left[\begin{array}{cc}
-0.1 & 0.01 \\
-0.2 & -0.1
\end{array}\right],
\end{aligned}
$$




$$
\begin{aligned}
& \rho_{1}=0.003, \\
& \rho_{2}=0.002 .
\end{aligned}
$$

The activation functions are taken as

$$
f_{1}(x)=f_{2}(x)=\tanh (0.5 x) .
$$

It can be verified that

$$
\begin{aligned}
L_{1} & =\left[\begin{array}{ll}
0 & 0 \\
0 & 0
\end{array}\right], \\
L_{2} & =\left[\begin{array}{cc}
0.25 & 0 \\
0 & 0.25
\end{array}\right] .
\end{aligned}
$$

In this example, if $\tau_{m}=3$ and $\tau_{M}=12$, the optimal passivity performance obtained is $\gamma=12.2439$ by the method in [25] and $\gamma=5.4007$ by the method in [26], while by Theorem 10 in this paper, the optimal passivity performance $\gamma=3.9546$. The comparisons of $\gamma$ are listed in Table 1 , when $\tau_{m}=3, \tau_{M}=$ $8,9,10,11,12$. Then, when we assume $\tau_{M}=13$, the optimal passivity performance $\gamma$ obtained by Theorem 10 for different $\tau_{m}$ can be found in Table 2 . It can be seen that our results are less conservative than the ones in $[25,26]$.

Example 2. Consider the system (1) with the following parameters:

$$
\begin{aligned}
A & =\left[\begin{array}{cc}
0.4 & 0 \\
0 & 0.5
\end{array}\right], \\
B_{1} & =\left[\begin{array}{cc}
0.001 & 0 \\
0 & 0.005
\end{array}\right], \\
B_{2} & =\left[\begin{array}{cc}
-0.1 & 0.01 \\
-0.2 & -0.1
\end{array}\right], \\
\rho_{1} & =\rho_{2}=0.003 .
\end{aligned}
$$

The activation functions are taken as

$$
f_{1}(x)=f_{2}(x)=\tanh (x) .
$$

It can be verified that

$$
\begin{aligned}
L_{1} & =\left[\begin{array}{ll}
0 & 0 \\
0 & 0
\end{array}\right], \\
L_{2} & =\left[\begin{array}{cc}
0.5 & 0 \\
0 & 0.5
\end{array}\right] .
\end{aligned}
$$

For this example, when $\gamma=3$ and $\tau_{m}=1$, by Theorem 10, we can get that the upper bound of the time-varying delay is $\tau_{M}=8$. When $\gamma=4$ and $\tau_{m}=1$, by Theorem 10, we can get $\tau_{M}=10$; we can obtain upper bound of $\tau_{M}$ for different $\gamma$ and $\tau_{m}$, which are summarized in Table 3 . It can be found from Table 3 that, for the same $\tau_{m}$, a larger passivity performance $\gamma$ corresponds to a larger $\tau_{M}$; with the same $\tau_{M}$, a smaller passivity performance $\gamma$ corresponds to a larger $\tau_{m}$.
TABLE 1: Optimal passivity performance $\gamma$ for different $\tau_{M}$.

\begin{tabular}{lccccc}
\hline Methods & 8 & 9 & 10 & 11 & 12 \\
\hline Theorem 1 [25] & 3.9708 & 4.8691 & 6.2501 & 8.3991 & 12.2439 \\
Corollary 2 & 2.9660 & 3.3483 & 3.8428 & 4.4968 & 5.4007 \\
[26] & & & & & \\
Theorem 10 & 2.8481 & 3.2154 & 3.5172 & 3.7438 & 3.9546 \\
\hline
\end{tabular}

TABLE 2: Optimal passivity performance $\gamma$ for different $\tau_{m}$.

\begin{tabular}{lccccc}
\hline Methods & 4 & 5 & 6 & 7 & 8 \\
\hline Theorem 1 [25] & 16.1853 & 13.2125 & 11.0130 & 9.2978 & 7.9134 \\
Corollary 2 & 6.1649 & 5.6743 & 5.2451 & 4.8664 & 4.5300 \\
[26] & & & & & \\
Theorem 10 & 4.4126 & 4.3752 & 4.2563 & 4.1871 & 4.0802 \\
\hline
\end{tabular}

TABLE 3: Allowable upper bounds of $\tau_{M}$ for different $\gamma$ and $\tau_{m}$.

\begin{tabular}{lccccc}
\hline$\tau_{m}$ & 1 & 3 & 5 & 7 & 9 \\
\hline$\gamma=3$ & 8 & 10 & 12 & 14 & 16 \\
$\gamma=4$ & 10 & 12 & 14 & 16 & 18 \\
$\gamma=6$ & 12 & 13 & 15 & 17 & 19 \\
\hline
\end{tabular}

\section{Conclusions}

In this paper, the problem of passivity analysis for discretetime stochastic neural networks with time-varying delays has been investigated. The presented sufficient conditions are based on the Lyapunov-Krasovskii functional, a new inequality and linear matrix inequality approach. Numerical examples are given to demonstrate the usefulness and effectiveness of the proposed results. Finally, it should be worth noticing that the proposed method in this paper may be extensively applicable in many other areas, such as Markov jump neural networks, Markov jump neural networks with incomplete transition descriptions, and switched neural networks, which deserves further investigation.

\section{Conflict of Interests}

The authors declare that there is no conflict of interests regarding the publication of this paper.

\section{Acknowledgments}

This work is supported by National Natural Science Foundation of China (Grants nos. 61273015 and 61473001), the Natural Science Research Project of Fuyang Normal College (2013FSKJ09), and the Teaching Reform Project of Fuyang Normal College (2013JYXM48).

\section{References}

[1] M. M. Gupta, L. Jin, and N. Homma, Static and Dynamic Neural Networks: From Fundamentals to Advanced Theory, John Wiley \& Sons, New York, NY, USA, 2003. 
[2] J. J. Hopfield, "Neurons with graded response have collective computational properties like those of two-state neurons," Proceedings of the National Academy of Sciences of the United States of America, vol. 81, no. 10 I, pp. 3088-3092, 1984.

[3] D. Yue, Y. Zhang, and E. Tian, "Improved global robust delay-dependent stability criteria for delayed cellular neural networks," International Journal of Computer Mathematics, vol. 85, no. 8, pp. 1265-1277, 2008.

[4] S. Xu, J. Lam, B. Zhang, and Y. Zou, "A new result on the delay-dependent stability of discrete systems with time-varying delays," International Journal of Robust and Nonlinear Control, vol. 24, no. 16, pp. 2512-2521, 2014.

[5] P.-L. Liu, "Delay-dependent global exponential robust stability for delayed cellular neural networks with time-varying delay," ISA Transactions, vol. 52, no. 6, pp. 711-716, 2013.

[6] P. L. Liu, "A delay decomposition approach to robust stability analysis of uncertain systems with time-varying delay," ISA Transactions, vol. 51, no. 6, pp. 694-701, 2012.

[7] J. Sun and J. Chen, "Stability analysis of static recurrent neural networks with interval time-varying delay," Applied Mathematics and Computation, vol. 221, pp. 111-120, 2013.

[8] D. Zhang and L. Yu, "Exponential state estimation for Markovian jumping neural networks with time-varying discrete and distributed delays," Neural Networks, vol. 35, pp. 103-111, 2012.

[9] P. G. Park, J. W. Ko, and C. Jeong, "Reciprocally convex approach to stability of systems with time-varying delays," Automatica, vol. 47, no. 1, pp. 235-238, 2011.

[10] C. Lin, Q.-G. Wang, and T. H. Lee, "A less conservative robust stability test for linear uncertain time-delay systems," IEEE Transactions on Automatic Control, vol. 51, no. 1, pp. 87-91, 2006.

[11] R. Lu, H. Wu, and J. Bai, "New delay-dependent robust stability criteria for uncertain neutral systems with mixed delays," Journal of the Franklin Institute. Engineering and Applied Mathematics, vol. 351, no. 3, pp. 1386-1399, 2014.

[12] J. Cheng, H. Zhu, S. Zhong, F. Zheng, and Y. Zeng, "Finite-time filtering for switched linear systems with a mode-dependent average dwell time," Nonlinear Analysis: Hybrid Systems, vol. 15, pp. 145-156, 2015.

[13] Z. Wang, G. Wei, and G. Feng, "Reliable $H_{\infty}$ control for discrete-time piecewise linear systems with infinite distributed delays," Automatica, vol. 45, no. 12, pp. 2991-2994, 2009.

[14] O. M. Kwon, M. J. Park, J. H. Park, S. M. Lee, and E. J. Cha, "New criteria on delay-dependent stability for discrete-time neural networks with time-varying delays," Neurocomputing, vol. 121, pp. 185-194, 2013.

[15] J. Cheng, H. Zhu, S. Zhong, Q. Zhong, and Y. Zeng, "Finitetime $H_{\infty}$ estimation for discrete-time Markov jump systems with time-varying transition probabilities subject to average dwell time switching," Communications in Nonlinear Science and Numerical Simulation, vol. 20, no. 2, pp. 571-582, 2015.

[16] T. Wang, C. Zhang, S. Fei, and T. Li, "Further stability criteria on discrete-time delayed neural networks with distributeddelay," Neurocomputing, vol. 111, pp. 195-203, 2013.

[17] X. Kan, H. Shu, and Z. Li, "Robust state estimation for discretetime neural networks with mixed time-delays, linear fractional uncertainties and successive packet dropouts," Neurocomputing, vol. 135, pp. 130-138, 2014.

[18] R. Lozano, B. Brogliato, O. Egeland, and B. Maschke, Dissipative Systems Analysis and Control: Theory and Applications, Springer, London, UK, 2nd edition, 2007.
[19] V. Bevelevich, Classical Network Synthesis, Van Nostrand, New York, NY, USA, 1968.

[20] B. Zhang, S. Xu, and J. Lam, "Relaxed passivity conditions for neural networks with time-varying delays," Neurocomputing, vol. 142, pp. 299-306, 2014.

[21] D. H. Ji, J. H. Koo, S. C. Won, S. M. Lee, and J. H. Park, "Passivity-based control for Hopfield neural networks using convex representation," Applied Mathematics and Computation, vol. 217, no. 13, pp. 6168-6175, 2011.

[22] Z. G. Wu, J. H. Park, H. Su, and J. Chu, "New results on exponential passivity of neural networks with time-varying delays," Nonlinear Analysis: Real World Applications, vol. 13, no. 4, pp. 1593-1599, 2012.

[23] T. H. Lee, M.-J. Park, J. H. Park, O.-M. Kwon, and S.-M. Lee, "Extended dissipative analysis for neural networks with timevarying delays," IEEE Transactions on Neural Networks and Learning Systems, vol. 25, no. 10, pp. 1936-1941, 2014.

[24] R. Raja, U. K. Raja, R. Samidurai, and A. Leelamani, "Passivity analysis for uncertain discrete-time stochastic BAM neural networks with time-varying delays," Neural Computing and Applications, vol. 25, no. 3-4, pp. 751-766, 2014.

[25] Q. Song, J. Liang, and Z. Wang, "Passivity analysis of discretetime stochastic neural networks with time-varying delays," Neurocomputing, vol. 72, no. 7-9, pp. 1782-1788, 2009.

[26] Z.-G. Wu, P. Shi, H. Su, and J. Chu, "Dissipativity analysis for discrete-time stochastic neural networks with time-varying delays," IEEE Transactions on Neural Networks and Learning Systems, vol. 24, no. 3, pp. 345-355, 2013.

[27] Z. Wu, P. Shi, H. Su, and J. Chu, "Stability and disspativity analysis of static neural networks with time delay," IEEE Transactions on Neural Networks and Learning Systems, vol. 23, no. 2, pp. 199-210, 2012. 


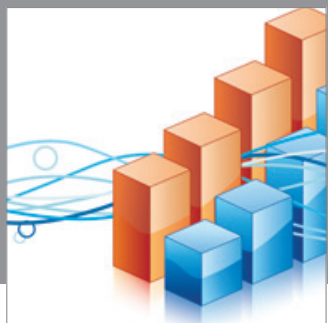

Advances in

Operations Research

mansans

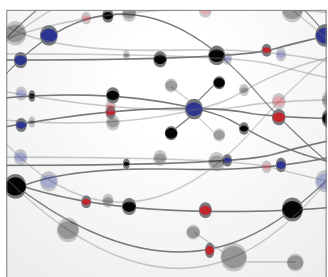

The Scientific World Journal
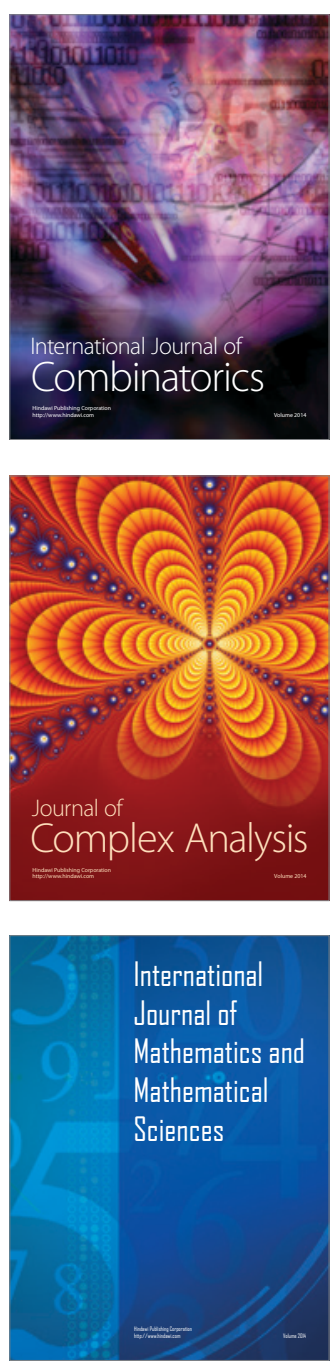
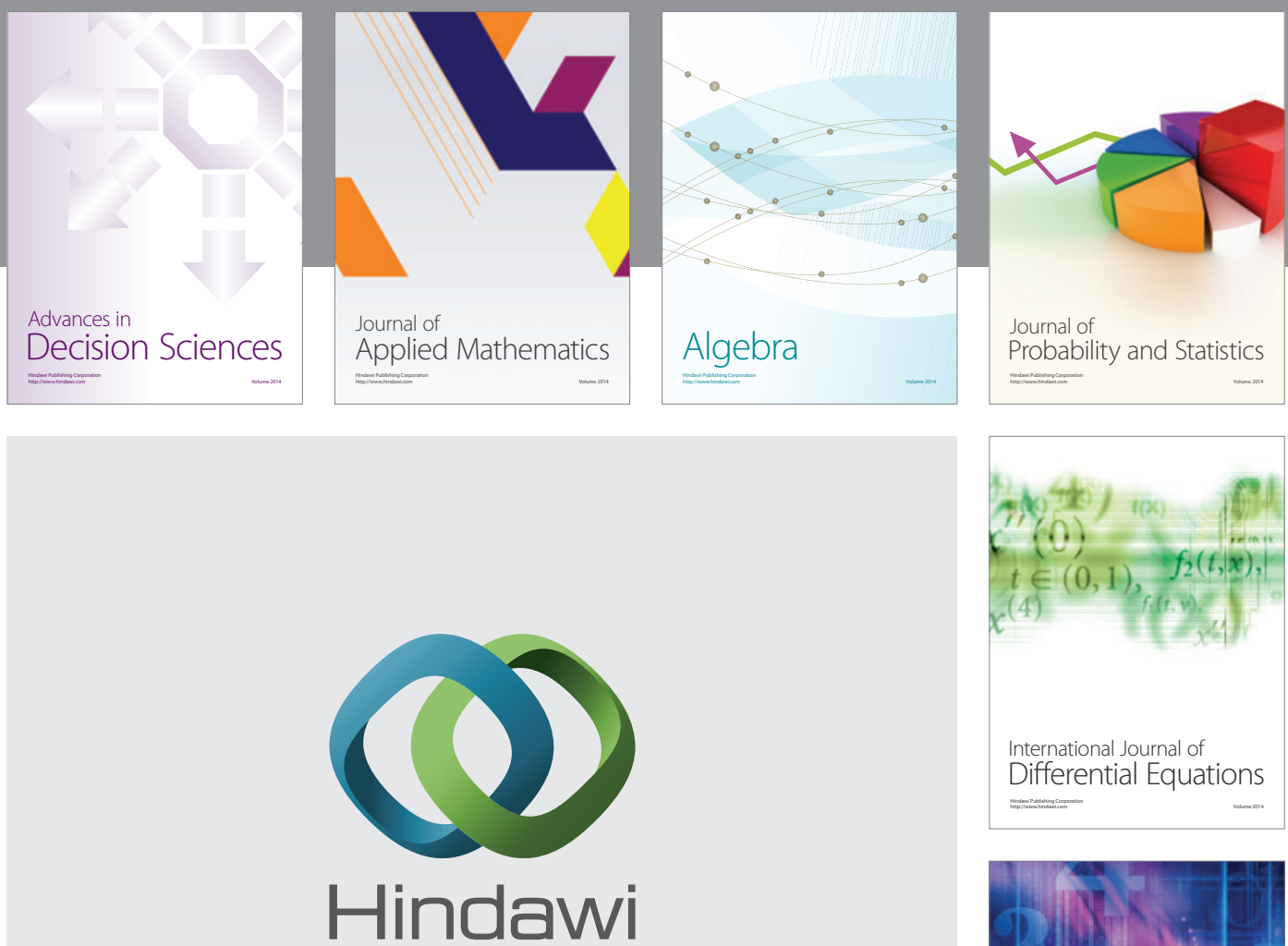

Submit your manuscripts at http://www.hindawi.com
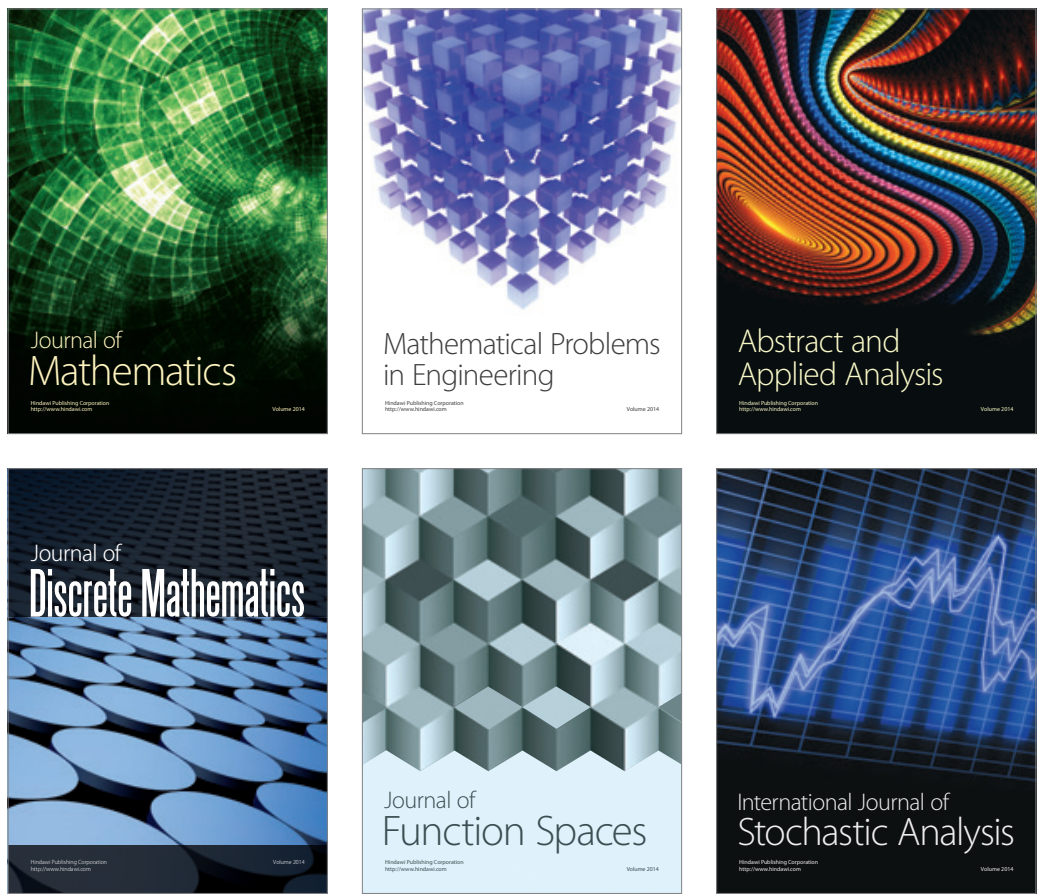

Journal of

Function Spaces

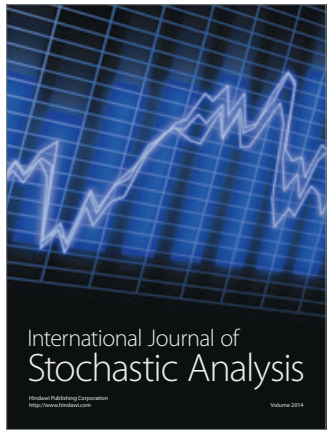

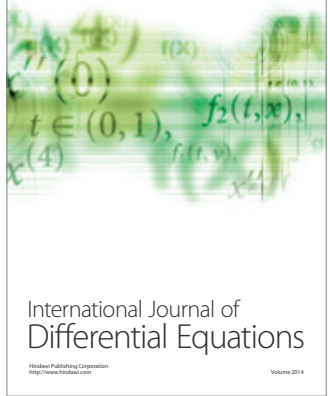
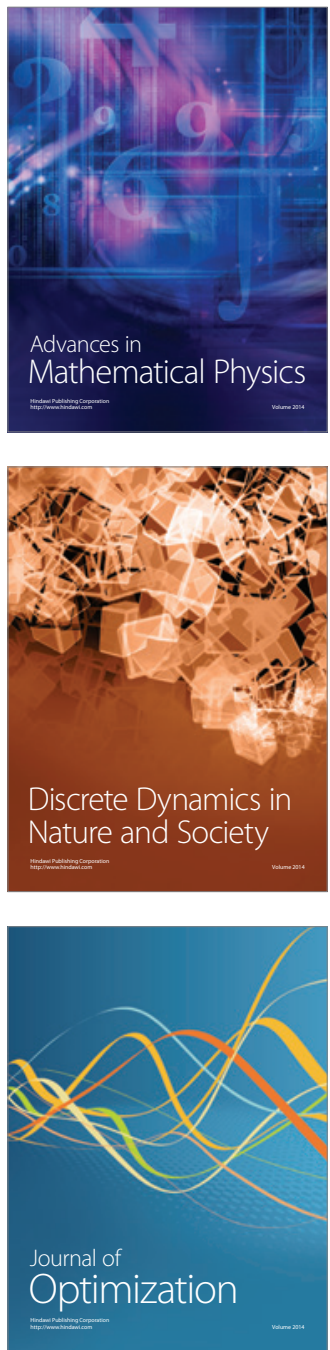